\title{
La formation des maîtres de géographie dans le canton de Vaud
}

\section{Introduction}

Le but de cet article est de présenter les grandes lignes de la formation pédagogique des maîtres de géographie dans le canton de Vaud. Le principe central de cette formation réside dans le fait qu'elle s'appuie sur un stage en responsabilité, ce qui permet au candidat d'être en prise directe sur la réalité de la classe tout au long de sa formation pédagogique initiale.

Il est utile de mentionner au préalable que dans le canton de Vaud toutes les classes des degrés 5 à 9 sont rattachées depuis 1986 au Service de l'enseignement secondaire. Au terme du degré 5 , qui est une année d'orientation, les élèves sont répartis en trois divisions: prégymnasiale, supérieure et terminale à options. Le personnel enseignant dans ces classes est composé à la fois de maîtres qui ont un titre universitaire et d'instituteurs formés dans les écoles normales. Le présent article se limite aux maitres licenciés, qui sont donc des spécialistes de branches, et qui enseignent surtout dans les divisions prégymnasiale et supérieure.

\section{Généralités}

Dans le canton de Vaud, la formation pédagogique initiale des maîtres de géographie licenciés s'effectue au Séminaire pédagogique de l'enseignement secondaire (SPES). Cette institution dépend du Service de l'enseignement secondaire supérieur et de la formation. Il n'existe pas de véritable lien institutionnel direct entre l'Université et le SPES. En pratique, aucune préparation spécifique à l'enseignement n'est d'ailleurs dispensée dans les facultés universitaires lausannoises. Toutefois, en ce qui concerne la géographie, les relations sont excellentes entre l'Institut de géographie de l'Université de Lausanne et les formateurs du SPES.

La clientèle du SPES est formée d'universitaires porteurs d'une licence agréée pour l'enseignement (en principe: lettres, sciences, mathématiques, HEC) ou d'un titre jugé équivalent. Les futurs maitres de dessin et de musique, qui reçoivent également leur formation pédagogique au SPES, doivent avoir obtenu un diplôme du Conservatoire ou de l'Ecole des Beaux-Arts, ou un titre équivalent. Tradition française oblige, les stagiaires qui suivent la didactique de la géographie sont des licenciés ès lettres, parfois des porteurs d'une licence HEC. En général, les stagiaires littéraires enseignent deux, voire trois branches. Pour les géographes, les combinaisons les plus fréquentes allient la géographie à l'histoire et/ou à une langue.

A l'heure actuelle, la formation pédagogique initiale dure une année. Cette formation méthodologique et didactique est combinée à un stage en responsabilité. Cela signifie que le maître stagiaire enseigne en principe 10 à 12 périodes hebdomadaires pendant toute l'année de formation, comme maitre pleinement responsable, dans des classes des degrés 5 à 9, ou du Secondaire supérieur (écoles professionnelles comprises). En dehors de l'enseignement qu'ils dispensent, les maitres stagiaires sont astreints à suivre des cours de psychopédagogie, de MAV, d'informatique et de didactique et méthodologie des branches qu'ils enseignent. Ceci représente en moyenne 12 à 14 heures hebdomadaires de cours.

Les formateurs, en particulier les responsables des didactiques de branches, visitent les stagiaires dans leurs classes à plusieurs reprises durant l'année (en moyenne 8 à 10 fois). Il faut noter ici que tous les formateurs de branche sont eux-mêmes actifs dans l'enseignement secondaire à côté de leur travail de méthodologues et de didacticiens. Deux formateurs animent la didactique de la géographie; ils sont engagés à $60 \%$, respectivement $50 \%$ d'un poste complet.

De 1986 à 1995 (période de fonction du signataire de l'article), 10 à 12 stagiaires ont en moyenne suivi la didactique de la géographie chaque année (pointe à 17 , minimum à 8). Problème particulier, la proportion des stagiaires porteurs d'une licence dont la géographie est l'une des branches a varie durant cette période entre 40 et $90 \%$ de la volée! En effet, un candidat peut être astreint à enseigner la géographie (ou d'autres branches) même s'il n'a pas de formation universitaire dans cette discipline! C'est le marché des places de stage disponibles qui peut dicter de telles situations: la formation pédagogique étant subordonnée à l'obtention d'une place de stage, les directeurs des établissements qui accueillent des stagiaires jouent un rôle important dans la définition du profil des places de stage; ce sont eux en premier lieu qui doivent veiller à l'adéquation de la place avec la formation académique du candidat.

Philippe Hertig, Séminaire pédagogique de l'enseignement secondaire, Avenue de Cour 33, 1007 Lausanne 
Comme il est ouvert à des personnes qui ont obtenu un titre universitaire, le SPES n'est en principe pas appelé à dispenser une formation scientifique. Mais les exceptions évoquées ci-dessus exigent que les formateurs assurent un appui adéquat aux stagiaires concernés; cet appui peut aller des conseils de lecture à l'apport direct des notions scientifiques nécessaires.

Au terme de la formation, le brevet est décerné si l'enseignement dispensé par le stagiaire est jugé suffisant sur l'ensemble de l'année pédagogique et si les conditions relatives aux cours et séminaires ont été remplies. Ce brevet permet à son titulaire de postuler pour une nomination dans tous les établissements de l'enseignement secondaire inférieur ou supérieur. Toutefois, à l'heure actuelle, ce sont les disciplines qui figurent sur le titre universitaire du candidat qui sont déterminantes en cas de postulation, et non celles qui ont été suivies au SPES (par exemple, un stagiaire littéraire licencié en français, allemand et histoire, et qui aurait suivi la didactique de la géographie au SPES tout en enseignant cette branche durant son année de stage, ne peut pas postuler pour une place de maître de géographie).

\section{Fonctionnement de la didactique de la géographie}

Quatre «piliers» contribuent à supporter l'édifice de la formation en didactique de la géographie: les séminaires de didactique (dont il sera question plus loin), les visites des formateurs dans les classes des stagiaires, les entretiens personnels entre stagiaires et formateurs, et le travail personnel (dossier) que doivent remettre les stagiaires.

Les visites dans les classes des stagiaires sont essentielles dans la mesure où elles permettent aux formateurs de se rendre compte de la qualité du contact entre le maître stagiaire et ses élèves, de sa capacité à gérer des situations relationnelles plus ou moins faciles ou difficiles, et de sa capacité à réaliser pratiquement un enseignement enrichissant pour ses élèves. Ces visites sont annoncées et sont toujours suivies d'une discussion entre le formateur et le stagiaire, discussion à but formatif.

Les entretiens personnels sont d'une importance comparable à celle des visites. Sous le terme "entretien» se cachent en fait des séances de travail consacrées soit à des problèmes concrets que le stagiaire rencontre momentanément, soit à des préparations en commun de sujets ou de leçons. C'est là une excellente occasion pour le formateur de comprendre la manière de travailler de chaque candidat et de l'aider, si nécessaire et dans la mesure du possible, à progresser dans sa manière de préparer son enseignement.

Enfin, le travail personnel demandé aux stagiaires consiste en un dossier qui présente un thème effectivement traité en classe, avec plan, démarche, documents, etc. Le but principal en est l'autoévaluation: il est demandé au stagiaire de porter une évaluation détaillée sur le travail en question. Il s'agit donc de faire une analyse cri- tique et raisonnée des qualités et des défauts du travail et de son succès (au sens d'efficacité et de réussite pédagogique) auprès des élèves.

\section{Les séminaires de didactique de la géographie}

L'année pédagogique s'articule en trois grandes phases pendant lesquelles l'accent est mis tour à tour sur des aspects différents de la didactique de la géographie. Toutes les séances sont conçues de manière à ce qu'elles soient un lieu d'échange, de mise en commun d'expériences vécues, d'idées et de matériel. La dotation horaire est actuellement de 4 périodes de 45 minutes à quinzaine.

Dans la première phase, après une séance visant à établir le contact dans le groupe de manière à ce qu'il bénéficie d'un climat de travail favorable, les séminaires sont consacrés à des notions de base d'utilité immédiate, appliquées à l'enseignement de la géographie, et dont les stagiaires ont besoin dès le début de leur enseignement. En partant des demandes des stagiaires motivées par leur pratique et en visant autant que possible la réalisation de matériel immédiatement utilisable en classe, les sujets suivants sont abordés, sur la base des principes de la pédagogie active: préparation et plan d'une leçon, plan à moyen terme, plan annuel, objectifs, niveaux d'activité des élèves, travaux écrits et évaluation. Cette première phase s'étend du début de l'année pédagogique aux vacances d'automne environ.

La seconde phase traite des principaux moyens et méthodes de l'enseignement de la géographie. Les stagiaires sont appelés à travailler sur des moyens tels que photographies et diapositives, croquis, vidéo (film), textes, schémas, graphiques, cartes, plans, etc. et sur des méthodes particulières telles que travaux de groupes, exposés d'élèves, jeux de rôles, jeux de simulation, travail dans le terrain, etc. En partant des besoins des stagiaires et en les mettant en situation, cette partie de l'année permet, grâce au passage en revue de ces différents moyens et méthodes d'enseignement, d'affiner les éléments abordés lors de la phase précédente, en particulier le plan de leçon, le plan à moyen terme et les objectifs de l'enseignement de la géographie. Cette période s'étend de novembre à fin janvier environ.

La troisième phase enfin est consacrée à des problèmes thématiques choisis parmi tous ceux que proposent les programmes officiels: géographie physique, géographie économique, géographie de la population, problèmes géographiques du Tiers-Monde, espaces menacés, géopolitique, aménagement du territoire: la liste est loin d'être exhaustive. C'est l'occasion de développer et d'approfondir les éléments traités précédemment et, surtout, de mener une réflexion sur les finalités de la discipline et de son enseignement (cette réflexion n'étant toutefois pas absente des phases précédentes!). Dans cette dernière partie de l'année pédagogique, qui va de février à fin mai, début juin environ, s'intercalent la présentation et l'analyse des travaux personnels des stagiaires. 
En raison de l'abondance des sujets qu'il serait possible de traiter et au vu de la diversité des programmes que les stagiaires sont appelés à enseigner, ce sont les demandes des stagiaires qui déterminent le programme des deuxième et troisième parties de l'année, tant pour l'énoncé des sujets retenus que pour l'ordre dans lequel ils sont abordés: un choix est nécessaire, et il est effectué par le groupe. Ainsi, seule la structure générale en trois grandes phases se retrouve d'une année à l'autre.

\section{Remarques conclusives}

Les formateurs du SPES sont unanimes à constater que la formule dans laquelle le stagiaire enseigne pendant sa formation représente un atout fondamental, mieux, une condition essentielle à la réussite d'une formation d'enseignant. En effet, la confrontation avec la pratique de la classe est en toutes circonstances le meilleur moyen d'expérimenter et de progresser dans l'enseignement.

Il faut néanmoins admettre que les stagiaires sans aucune expérience préalable de l'enseignement peuvent connaître de sérieuses difficultés en début d'année. Ils sont en tous les cas confrontés à une multitude de questions nouvelles en même temps, ce qui peut être un facteur de stress non négligeable. Par ailleurs, certains stagiaires se trouvent dans des situations de stage pénibles (classes difficiles, enseignement d'une branche sans avoir la formation académique, ou encore encadrement insuffisant dans l'établissement de stage, par exemple); de telles situations n'assurent sans doute pas des conditions de formation satisfaisantes.

Avec une dotation globale de 72 à 80 périodes environ sur l'ensemble de l'année pour la didactique de la géographie, la formation initiale est très courte... Il se pose alors le problème de la formation continue! Celle-ci reste pour l'essentiel tributaire de l'engagement et de la volonté propre de chaque enseignant. Les possibilités et facilités accordées aux maîtres licenciés vaudois dans ce domaine devraient impérativement être améliorées. De même, les liens entre formation initiale et formation continue sont largement perfectibles.

Au-delà de ces remarques qui ne concernent pas que la géographie, je souhaite conclure en insistant sur l'importance cruciale que revêt aujourd'hui la formation dans notre pays. C'est vrai à tous les niveaux, de l'école primaire à l'université comme dans toutes les filières de formation professionnelle. Au risque de recourir à un cliché, je rappellerai que la Suisse n'a pratiquement pas d'autres ressources que sa «matière grise». A l'heure où tout le système de formation suisse s'apprête à vivre de sérieuses mutations (réorganisation de systèmes scolaires cantonaux, nouvelle ORM, apparition des HEP et des HES, etc.), il serait grand temps que le monde politique comprenne qu'il n'y a pas de plus grave erreur que de menacer la qualité de la formation en Suisse pour des raisons budgétaires.

En ce qui concerne les enseignants, le défi est triple:

- la formation scientifique de base se doit d'être excellente; je serais à cet égard tenté d'exiger que tous les futurs enseignants fassent des études de type universitaire (sans qu'il y ait forcément l'obligation d'un parcours académique complet pour toutes les catégories d'enseignants);

- la formation pédagogique initiale doit préparer les futurs maîtres aux exigences des écoles d'aujourd'hui et de demain;

- la formation continue doit offrir les nécessaires ressourcements dont les enseignants ont besoin tout au long de leur carrière.

Sur ces trois plans, la géographie peut être considérée comme une discipline emblematique. Une solide formation de base de type universitaire est indispensable pour se familiariser avec la géographie actuelle, qui n'est plus la discipline descriptive qu'elle était voici quelques décennies encore. Au cours de sa formation pédagogique initiale, l'enseignant acquiert les outils didactiques et méthodologiques qui lui permettent de faire de la géographie scolaire une discipline-clé dans le bagage culturel et scientifique de ses élèves. Enfin, la formation continue assure le prolongement, le renouvellement et le perfectionnement des deux précédentes. 